\title{
Adapting to a novel disruptive threat: Nuclear Cardiology Service in the time of the Coronavirus (COVID-19) Outbreak 2020 (SARS REBOOT)
}

\author{
Kelvin S. H. Loke, MBBS, MRCP, ${ }^{\text {a,d }}$ Wei Ying Tham, MBBS, MRCP, ,d \\ Pushan Bharadwaj, MBBS, FRCP, FEBNM, DipCBNC, ,d Felix Y. J. Keng, MBBS, \\ FRCP, ${ }^{\text {b,d }}$ Zijuan Huang, MBBS, MRCP, ${ }^{\text {b }}$ Muhammad Bin Idu, MBBS, MRCP, ${ }^{\text {b,d }}$ \\ Yen Ming Wong, Nitec, ${ }^{\mathrm{C}}$ Paul Kia Siong Tan, BSci, ${ }^{\mathrm{c}}$ Sumbul Zaheer, MBBS, \\ MRCP, a,d Yiu Ming Khor, MBBS, MRCP, a,d David Chee Eng Ng, MBBS, MSc, \\ FRCP, ${ }^{\text {a,d }}$ Wai Yin Wong, MBBS, FRCR, ${ }^{\text {a,d }}$ and Aaron Kian-Ti Tong, MBBS, MMed, \\ $M R C P^{\mathrm{a}, \mathrm{d}}$ \\ a Department of Nuclear Medicine and Molecular Imaging, Singapore General Hospital, \\ Singapore, Singapore \\ b Department of Cardiology, National Heart Centre Singapore, Singapore, Singapore \\ c Department of Radiology, Sengkang General Hospital, Singapore, Singapore \\ d Duke-NUS Medical School, Singapore, Singapore
}

Received Mar 21, 2020; accepted Mar 23, 2020

doi: $10.1007 / \mathrm{s} 12350-020-02117-0$

\section{INTRODUCTION}

China alerted the World Health Organization (WHO) to several cases of unusual pneumonia in the city of Wuhan, Hubei Province in central China on 31st December 2019. ${ }^{1,2}$ The causative agent was reported to be a new type of coronavirus by 7 th January 2020 . Within the same month, the lockdown of several Hubei cities was instituted by China as cases increased rapidly. Imported cases of coronavirus infection were soon detected in several neighboring countries including South Korea, Japan, Thailand, and Singapore in the same month. The virus continued to spread to other provinces of China and worldwide.

The first case of imported coronavirus in Singapore was identified on 23 January 2020. In the subsequent days, the number of new cases continued to rise and at

\footnotetext{
Electronic supplementary material The online version of this article (https://doi.org/10.1007/s12350-020-02117-0) contains supplementary material, which is available to authorized users.

The authors of this article have provided a PowerPoint file, available for download at SpringerLink, which summarises the contents of the paper and is free for re-use at meetings and presentations. Search for the article DOI on SpringerLink.com.

Reprint requests: Kelvin S. H. Loke, MBBS, MRCP, Department of Nuclear Medicine and Molecular Imaging, Singapore General Hospital, Singapore, Singapore; kelvin.loke.s.h@singhealth.com.sg J Nucl Cardiol 2020;27:1005-9. $1071-3581 / \$ 34.00$

Copyright (C) 2020 American Society of Nuclear Cardiology.
}

one point of time, Singapore had the highest number of infected cases worldwide outside China. On 11 February 2020, WHO announced that the disease caused by the virus (SARS-CoV-2) would be called COVID-19. One month later on 12th March 2020, WHO declared COVID-19 a global pandemic. At the latest count on 18th March 2020, the number of confirmed infected cases worldwide has exceeded 209,503 in 150 countries with cases reported in all continents except Antarctica and the global reported death toll has risen to 8,784 .

The Ministry of Health in Singapore raised the Disease Outbreak Response System Condition (DORS$\mathrm{CON}$ ) to orange, the second highest level, within 15 days of the first reported case of COVID-19 in Singapore in response to the increased risk of community spread of disease. Since then, Singapore has seen 313 confirmed cases of COVID-19 (mostly imported cases) and no resultant deaths to date, with early detection and containment being currently the overall strategy to combat this disease on a whole-of-government approach. ${ }^{3}$ Medical institutions have had to rapidly adapt in tandem with governmental policies and the key imperatives identified are to treat suspect and confirmed cases while paying attention to avoid intra-hospital and cross-institutional transmission between patients and staff.

A previous editorial summarized the challenges for Nuclear Medicine Departments in this COVID-19 
pandemic. ${ }^{4}$ In this short communication sharing our current experience in Singapore, the key considerations were for an adapted Nuclear Cardiology Service, particularly within an institutional/hospital and hospital group setting, to prepare in terms of policies and processes with the focused aim of avoiding intrainstitutional and cross-institutional spread of infection while being nimble enough to adopt new practice changes in the rapidly evolving COVID-19 outbreak situation. Drawing on our past experiences and lessons learnt during the Severe Acute Respiratory Syndrome (SARS) coronavirus crisis in 2003, our overall response to this COVID-19 situation was rapid and decisive. Many of these policies and processes are common to the institution and radiological services and also dedicated nuclear cardiology practices.

\section{CONSIDERATIONS OF POLICIES AND PROCESSES}

As a background to this write-up, our hospital group comprises a network of acute hospitals, national specialty centers (for example the Singapore General Hospital, National Heart Centre, National Dental Centre, Singapore National Eye Centre etc.), community hospitals and polyclinics providing services ranging from primary to quaternary care to serve the healthcare needs of the central, eastern and northeastern parts of Singapore. In addition, the SingHealth Duke-NUS academic medical center is also physically located within the SingHealth Outram main campus.

Nuclear cardiology services reside within these tertiary/quaternary institutions located in the aforementioned locations within Singapore and are supported by both nuclear cardiologists and nuclear medicine physicians. There are 4 dedicated cardiac gamma cameras ( 2 of which are CZT detector cameras) in the National Heart Centre whilst Singapore General Hospital has 4 gamma cameras (1 SPECT/CT) and 1 PET/CT scanner. The types of scans performed include myocardial perfusion imaging (MPI) and advanced imaging with PET tracers with the total annual workload averaging 10,000 scans or more.

\section{General Infection Control Measures}

The key to avoiding disease transmission of a relatively new and unknown infectious agent, is the swift adoption of strict personal and institutional infection control practices appropriate to the overall situation, as well as the ability to stratify patients according to their risk of COVID-19 infection.

Proper degrees of masking (surgical mask, N95 mask, eye protection) and other personal protective equipment (gowns, gloves) were quickly identified for doctors and staff handling different risk groups of patients and different categories of procedures. As evidence suggests that the SARS-CoV-2 virus was spread via droplets, procedures that involved patient coughing or otherwise producing droplets or aerosols were considered higher risk procedures.

Training sessions were conducted for staff on the proper use of surgical or N95 masks and various personal protective equipment. Staff who failed N95 mask training on at least two separate occasions were trained in donning Powered Air Purifying Respirator (PAPR) so that every individual in the team would remain operationally ready for cases.

Hand hygiene was emphasized and frequent sanitization of hands and work surfaces were reinforced. Regular audits and management walkabouts helped quickly identify lapses in infection control practices which were promptly communicated to the department/division. Sharing of good clinical and infection control practices served to boost staff morale as COVID19 pandemic was deemed to last for months.

\section{Screening of Patients and Visitors}

Screening of patients and visitors as they arrive in the department is critical. Typical screening methodology included recording relevant personal particulars (to facilitate contact tracing), taking a targeted history of travel, detailed contact history with any known clusters of disease, screening of electronic medical records as well as any flulike respiratory symptoms. Temperature taking at entrances using mass screening thermal imaging devices was the first line to identify suspect patients, although it is also known that not all infected patients are symptomatic nor febrile. In an attempt to further reduce risks, the number of caregivers allowed to accompany patients was limited to 1 per patient and social distancing was practiced in the waiting areas by ensuring that every other seat was left empty.

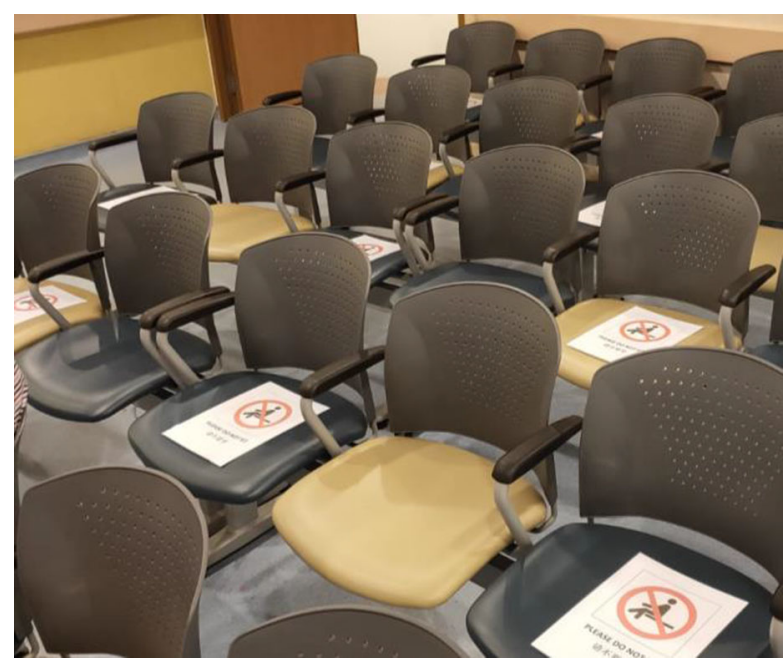

Picture of patient waiting room. 
An isolation room had to be designated to temporarily hold any suspect cases of the infection who may have arrived at the department inadvertently. The purpose of this isolation room was to provide a safe area away from other patients and visitors, where suspect cases can be reviewed by a physician for further management.

\section{Segregation of Risk Groups and Conduct of MPI}

Next, the segregation of high-risk vs low-risk patients in terms of suspicion of COVID-19 serves to reduce the chance of intra-institutional spread of disease as well as to facilitate or simplify contact tracing. It is also imperative to consider the indications and urgency for MPI during this pandemic. Referring physicians should discuss and justify the urgency of the procedure with a nuclear cardiologist or physician if an MPI is ordered for confirmed or suspected COVID-19 patients to reduce unnecessary exposure of healthcare workers to risks of infection.

The ideal clinical setup would be to have duplicate clinical interview and treadmill rooms as well as duplicate gamma camera systems which will allow complete physical separation of high and low-risk patients. However, such a perfect system hardly exists due to the tremendous manpower and financial resources required. Instead, physical or temporal segregation is a more realistic option. Physical segregation involves defining boundaries to dichotomize the clinical area into high and low-risk zones where patient movement are strictly confined to their appropriate risk levels versus temporal separation, where patients were separated in terms of time.
Temporal segregation is particularly useful in facilities that are constrained in terms of physical layout of the department and limited number of scanners, such as in our Department which is a busy nuclear medicine facility seeing an admixture of oncology, endocrinology, rheumatology and cardiology patients where a myriad of both diagnostic and therapeutic nuclear medicine procedures are performed, not limiting to nuclear cardiology alone.

Thus, we managed our myocardial perfusion imaging workflow (MPI) as follows:

- Within the Outram campus, a decision was made to separate outpatient from inpatient referrals. Nuclear cardiology examinations for outpatients were conducted in the National Heart Centre, while inpatients underwent their examinations in the Singapore General Hospital.

- In the satellite acute hospitals, outpatients were strictly separated temporally from inpatients. As far as possible, patient referrals were batched into groups (inpatient/outpatient) and selected days in the week were allocated to either group without overlap.

- On outpatient days when an urgent inpatient MPI was required, the inpatients and outpatients were separated physically and temporally. The inpatients were stressed and scanned separately and preferably after completing the outpatient scans for the day with terminal cleaning in between.

Exercise stress testing for myocardial perfusion imaging was identified as a high-risk procedure for droplet production. As such, treadmill exercise stress was discouraged over pharmacological stress and med$\mathrm{ical} /$ nursing staff who attended to suspect patients were required to don N95 masks with appropriate PPE. 


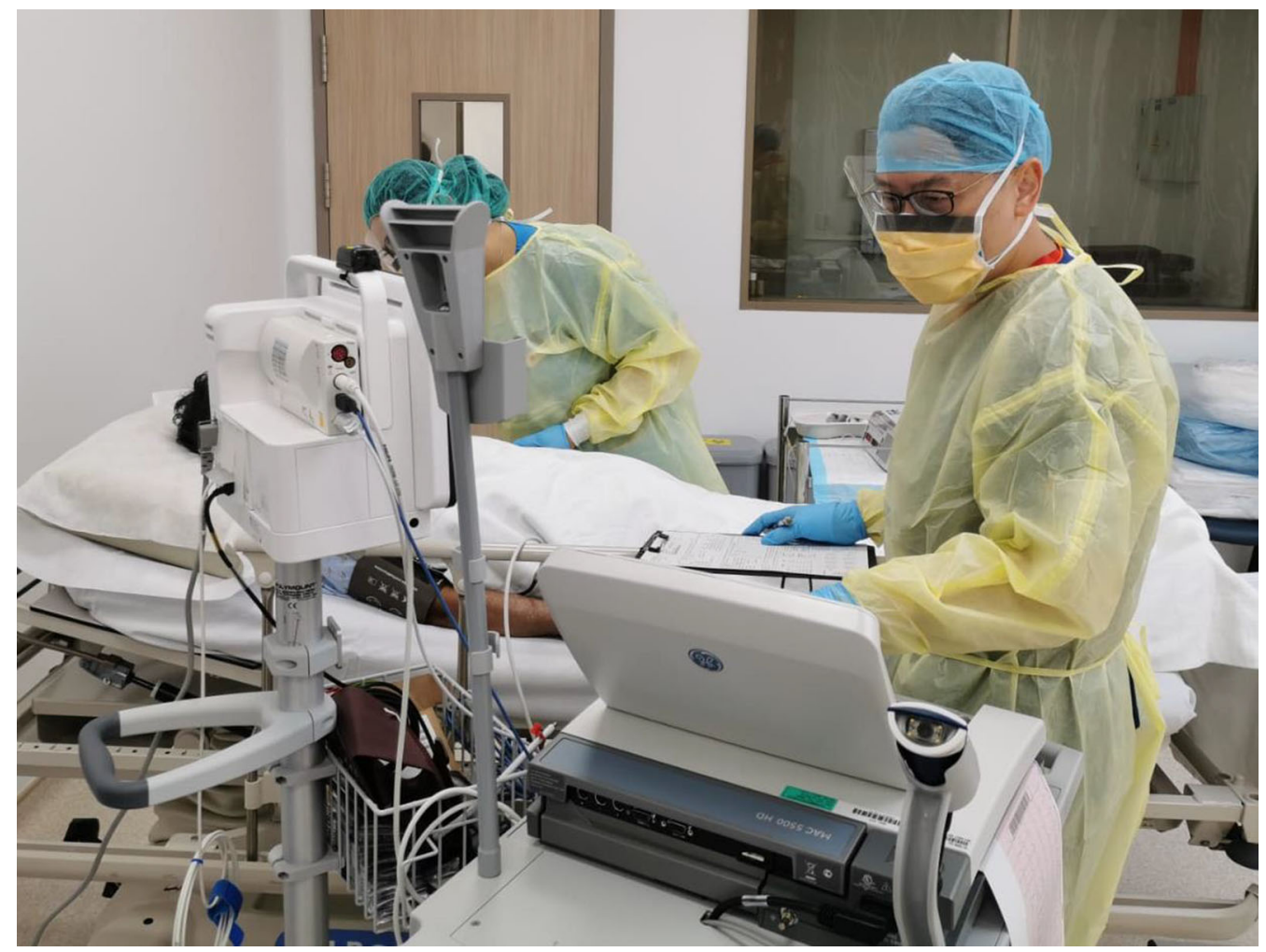

Donning appropriate PPE for pharmacologic stress testing in COVID-19 status unknown patients or suspected cases.

Patients' time spent in the department was minimized as far as possible; for suspected COVID-19 cases, a switch (from the usual stress-rest protocol) to a rest scan followed by pharmacologic stress scan was adopted, keeping the waiting time between rest and stress imaging to a minimum.

Following publications on the chest CT findings in COVID-19 patients $^{5-7}$, we instituted a new requirement for the $\mathrm{CT}$ (acquired for attenuation correction or as part of the PET/CT scan) images to be screened before allowing patients to leave the department. This provides the opportunity to promptly identify asymptomatic patients with CT changes suspicious for COVID-19, such as ground-glass opacities in a peripheral distribution and alert the referring clinician for further management.

\section{Segregation of Staff and Service Continuity Plans}

Staff can be infected and become a nidus for infection within the hospital, potentially leading to a massive shutdown of the service if no contingency plans are afoot. As was practiced during the time of SARS in $2003,{ }^{8}$ our clinical staff were segregated into 2 teams, who worked and rested in alternate shifts of 2 or more weeks, so that in the event of any contact with an unexpected case, the affected team can stand down, while the other team takes over.

Staff were mandated to monitor and $\log$ their temperature and location twice daily as a surveillance for early sign of potential infection and were encouraged to practice social distancing.

Given the possibility of virus survival and transmission via fomites, the rapidity and spread of the virus to many countries around the globe as well as the increased precautions taken, we had also noted diminishing stocks of pharmaceuticals commonly used in nuclear cardiology service. As such, there should also be awareness towards essential equipment (surgical and N95 masks etc.) and medication stocks during this period especially in large tertiary referral centers like ours with a high throughput of patients.

\section{SUMMARY}

The COVID-19 pandemic has rapidly swept across the globe from Wuhan, China to the rest of the world in a matter of weeks with exponentially increasing numbers of infected patients and fatalities worldwide. Health 
experts have warned that this pandemic may last for months up to a year. Tapping on past experiences from the SARS coronavirus epidemic of 2003, we have swiftly implemented the various policies and procedures to adapt our workflow to meet this novel disruptive threat whilst continuing to provide essential nuclear cardiology service in the local population. With these measures in place and strict adherence to our infection control protocols, there has not been any patient to physician transmission of COVID-19 within the local nuclear cardiology departments to date.

\section{Disclosures}

The authors have no disclosures to declare.

\section{References}

1. Zhu N, Zhang D, Wang $\mathrm{W}$ et al. A novel coronavirus from patients with pneumonia in China, 2019. N Engl J Med 2020;382:727-33.
2. Huang C, Wang Y, Li X et al. Clinical features of patients infected with 2019 novel coronavirus in Wuhan, China. Lancet 2020;395:497-506.

3. Wong JEL, Leo YS, Tan CC. COVID-19 in Singapore-current experience. Critical global issues that require attention and action. JAMA 2020. https://doi.org/10.1001/jama.2020.2467.

4. Huang HL, Allie R, Gnanasegaran G, Bomanji J. COVID-19Nuclear Medicine Departments, be prepared! Nucl Med Commun 2020. https://doi.org/10.1097/MNM.0000000000001183.

5. Ai T, Yang Z, Hou $\mathrm{H}$ et al. Correlation of chest $\mathrm{CT}$ and RT-PCR testing in Coronavirus Disease 2019 (COVID-19) in China: A report of 1014 cases. Radiology 2020. https://doi.org/10.1148/rad iol.2020200642.

6. Xu X, Yu C, Qu J et al. Imaging and clinical features of patients with 2019 novel coronavirus SARS-CoV-2. Eur J Nucl Med Mol Imaging 2020. https://doi.org/10.1007/s00259-020-04735-9.

7. Qin C, Liu F, Yen T et al. ${ }^{18}$ F-FDG PET/CT findings of COVID-19: a series of four highly suspected cases. Eur J Nucl Med Mol Imaging 2020. https://doi.org/10.1007/s00259-020-04734-w.

8. Tsou IYY, Goh JSK, Kaw GJL et al. Severe acute respiratory syndrome: Management and reconfiguration of a radiology department in an infection disease situation. Radiology 2003;229:21-26.

Publisher's Note Springer Nature remains neutral with regard to jurisdictional claims in published maps and institutional affiliations. 